\title{
Surface-enhanced Raman spectroscopic study of DNA and 6-mercapto-1-hexanol interactions using large area mapping
}

Frøhling, Kasper Bayer; Alstrøm, Tommy Sonne; Bache, Michael; Schmidt, Michael Stenbæk; Schmidt, Mikkel Nørgaard; Larsen, Jan; Jakobsen, Mogens Havsteen; Boisen, Anja

Published in:

Vibrational Spectroscopy

Link to article, DOI:

10.1016/j.vibspec.2016.08.005

Publication date:

2016

Document Version

Peer reviewed version

Link back to DTU Orbit

Citation (APA):

Frøhling, K. B., Alstrøm, T. S., Bache, M., Schmidt, M. S., Schmidt, M. N., Larsen, J., Jakobsen, M. H., \& Boisen, A. (2016). Surface-enhanced Raman spectroscopic study of DNA and 6-mercapto-1-hexanol interactions using large area mapping. Vibrational Spectroscopy, 86, 331-336.

https://doi.org/10.1016/j.vibspec.2016.08.005

\section{General rights}

Copyright and moral rights for the publications made accessible in the public portal are retained by the authors and/or other copyright owners and it is a condition of accessing publications that users recognise and abide by the legal requirements associated with these rights.

- Users may download and print one copy of any publication from the public portal for the purpose of private study or research.

- You may not further distribute the material or use it for any profit-making activity or commercial gain

- You may freely distribute the URL identifying the publication in the public portal 


\title{
Surface Enhanced Raman Spectroscopic Study of DNA and 6- mercapto-1-hexanol Interactions Using Large Area Mapping
}

Kasper Bayer Frøhling ${ }^{\mathrm{a} \dagger}$, Tommy Sonne Alstrøm ${ }^{\mathrm{b}}$, Michael Bache ${ }^{\mathrm{a}}$, Michael Stenbæk Schmidt ${ }^{\mathrm{a}}$, Mikkel Nørgaard Schmidt ${ }^{\mathrm{b}}$, Jan Larsen ${ }^{\mathrm{b}}$, Mogens Havsteen Jakobsen ${ }^{\mathrm{a}}$, Anja Boisen ${ }^{\mathrm{a}}$

${ }^{\text {a }}$ Technical University of Denmark, Department of Micro- and Nanotechnology, Ørsteds Plads building 345Ø, 2800 Kgs. Lyngby, Denmark.

${ }^{\mathrm{b}}$ Technical University of Denmark, Department of Applied Mathematics and Computer Science, Richard Petersens Plads building 324, 2800 Kgs. Lyngby, Denmark.

${ }^{\dagger}$ Corresponding author: kasper.frohling@gmail.com

\begin{abstract}
The emergence of 2D SERS substrates with large areas of hot spots has enabled data to be gathered at large scale. This work presents a statistical tool for analysing large amounts of SERS data by utilizing a peakfitting model in a specific spectral range. By analysing the distributions of Raman intensities and peak positions it is possible to directly inspect the interplay between DNA and 6-mercapto-1-hexanol on gold covered nanopillars. It is demonstrated that optimised functionalization parameters can be extracted from the Raman spectra directly. Using the peak-fitting approach it is possible to avoid miss-interpretation of intensity histograms, where contamination might contribute with an enhanced background and not a peak.
\end{abstract}

Keywords: SERS, area mapping, DNA, 6-mercapto-1-hexanol, thiol, gold, peak-fitting.

\section{1}

\section{Introduction}

Surface Enhanced Raman Spectroscopy (SERS) is a sensitive technique for detection of molecules. Based on inelastic scattering of light and high enhancement of electromagnetic fields SERS has been used for single molecule detection of many substances [1-4]. Traditionally, SERS has been achieved by clustering of silver or gold nanoparticles [5,6], but recent advances in nanotechnology have enabled fabrication of SERS substrates with 2D arrays of homogeneous nanostructures suitable for electromagnetic field enhancement. In the vast majority of nanoparticle SERS studies only few $(\mathrm{n}<10)$ Raman spectra are recorded and analysed. This is due to the inherent nature of nanoparticle clusters, which only form in small areas on solid supports. The development of 2D substrates with large areas of hot spots has enabled an easy way to obtain statistically significant amounts of data. The distribution of SERS intensities arising from hot spots varies greatly due to the highly localised electromagnetic field enhancement factors between metal surfaces in close proximity [5]. Etchegoin et al. [7] argue that the statistical behaviour of SERS intensities follow a long/heavy tailed distribution, which has likewise been observed by other groups $[8,9]$.

In this study we report on the statistical analysis of large area mapping of SERS data $(n=300)$ collected from an in-house developed 2D SERS substrate. We investigate the interplay between thiolated DNA and 6mercapto-1-hexanol (MCH) on a gold covered silicon nanopillar surface using a developed peak-fitting 
model. The interaction between self-assembled monolayers (SAMs) of MCH and immobilised DNA strands on silver or gold surfaces has been widely used in biosensing assays [9-12]. MCH helps maintaining the biological activity and availability of the DNA strand by blocking remaining gold binding sites, thus preventing unspecific binding. Unspecific binding of unwanted molecules and of DNA sugar phosphate backbone is prevented by MCH SAMs. In this study we argue that optimised functionalization parameters of the gold nanopillars can be found directly by inspecting the combined spectra of DNA and MCH. To achieve this we use a peak-fitting model on the 300 spectra in each sample.

\section{2}

\section{Experimental}

2.1

\section{Materials}

A 75-mer DNA strand was purchased from DNA Technology A/S (Aarhus, Denmark) with the sequence: 5'-ATA CGA GCT TGT TCA ATA CGA AGG GAT GCC GTT TGG GCC CAA GTT CGG CAT AGT GTG GTG ATA GTA AGA GCA ATC-3'. It was modified with a $\mathrm{C}_{6}$-S-S linker in the 5'-end. Illustra MicroSpin G-25 Columns (27-5325-01, GE Healthcare) were used for purification of the DNA. Molecular biology grade water (Sigma-Aldrich), $99 \%$ ethanol (Sigma-Aldrich), 1x Phosphate Buffered Saline (1xPBS) (Sigma-Aldrich), TCEP•HCl (Pierce) and 6-mercapto-1-hexanol (TCI Chemicals) were all used as received. Eppendorf DNA LoBind ${ }^{\mathrm{TM}}$ micro tubes and LoRetention ${ }^{\mathrm{TM}}$ pipette tips were used when appropriate.

\section{2}

\section{Fabrication of SERS substrate}

Gold-coated silicon nanopillars were fabricated in accordance with previously published methods [13]. A 4" silicon wafer $(<100>$, single-side polished, boron doped) was etched for $4 \mathrm{~min}$ in a mask-less Reactive Ion Etch (RIE) (STS ICP Advanced Silicon Etcher, SPTS Technologies) at $-10^{\circ} \mathrm{C}$ followed by a 1 min $\mathrm{O}_{2}-$ plasma cleaning procedure. This created randomly distributed silicon nanopillars, which were subsequently covered with app. $200 \mathrm{~nm}$ gold using e-beam evaporation (Alcatel SCM 600: $8 \mathrm{kV}$ acceleration voltage, 10 $\AA / \mathrm{s}$ deposition rate). The nanopillars had height and diameter of about $500 \mathrm{~nm} \times 100 \mathrm{~nm}$. After fabrication, the wafer was diced into smaller pieces (roughly $4 \mathrm{~mm} \times 4 \mathrm{~mm}$ ). When the nanopillars were dried from a wet state, they formed small clusters of leaned pillars where the hot spots are created. This SERS substrate is reliable for creating reproducible $2 \mathrm{D}$ arrays of electromagnetic hot spots with similar enhancement factors $[13,14]$.

\section{3}

\section{Surface functionalization}

Enhancement of the Raman signal happens between the narrow gaps of metal surfaces. On this substrate these spots occur when pillars lean towards each other, creating a narrow gap between two gold heads. Therefore molecules of interest must be caught in these hot spots. Since the DNA has a disulphide (S-S) modification it is possible to target the gold selectively to the silicon by breaking this bond into individual thiol (-SH) bonds. All incubation steps were performed without stirring and at room temperature. The steps of the functionalization procedure are shown schematically in Figure 1. Firstly, the surface is cleaned (Figure 1a) to remove organic contaminants and enhance wettability followed by DNA immobilization (Figure 1b/c) and MCH backfilling (Figure 1d). 


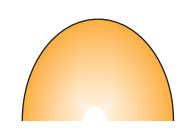

a

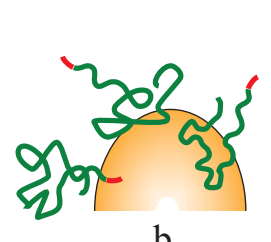

Fast

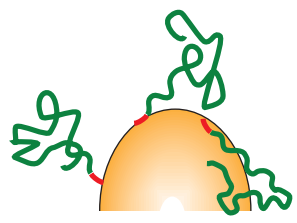

c

Rate-limiting step

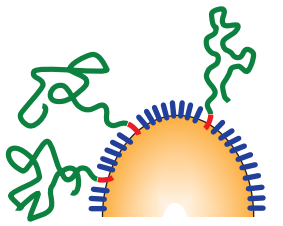

d

Fast

NDNA strand - Thiol group -6-mercapto-1-hexanol

Figure 1: Schematic representation of the functionalization protocol. The thiolated DNA strand is firstly physically adsorbed to the clean nanopillar gold surface $(a, b)$. Further incubation allows the thiol group to form a covalent-like bond to the gold (c), which is the rate-limiting step. Backfilling with 6-mercapto-1-hexanol lifts the DNA from the surface and blocks the remaining gold binding sites (d) to prevent unspecific binding ensuring complete functionality of the DNA.

\subsection{1}

\section{Pre-treatment and cleaning of substrate}

Prior to any surface functionalization the chips were cleaned in $\mathrm{O}_{2}$-plasma for 35 seconds at 0.5 mbar oxygen pressure (Plasma cleaner ATTO, Diener Surface Technology) followed by immersion in ethanol and $\mathrm{H}_{2} \mathrm{O}$ respectively ( $3 \mathrm{~min}$ each). This removed any organic contaminants that might have accumulated since fabrication, and at the same time made the gold surface hydrophilic. From this step onwards the chips were not allowed to dry until the final step, since this would have facilitated irreversible leaning of the nanopillars, making it difficult to trap molecules in hot spots.

\subsection{2}

\section{Conjugation of DNA to Au nanopillars}

The disulphide bond of the DNA 5'-modification was reduced to two individual thiol (-SH) functional groups by mixing $100 \mu \mathrm{l}\left(5 \mu \mathrm{M}\right.$ in $\left.\mathrm{H}_{2} \mathrm{O}\right)$ DNA solution with $100 \mu \mathrm{l}\left(500 \mu \mathrm{M}\right.$ in $\left.\mathrm{H}_{2} \mathrm{O}\right)$ TCEP•HCl solution. After 60 min incubation the solution was purified on Illustra MicroSpin G25 columns to remove small nonDNA molecules. The cleaned, wet chips were immersed into the purified solution after addition of $300 \mu 1$ $1 \times$ PBS and incubated with varying incubation times ( $0 \mathrm{~min}$ to $240 \mathrm{~min})$. The final DNA concentration was 1 $\mu \mathrm{M}$.

\subsection{3}

\section{Backfilling with MCH}

The chips were transferred directly to the $\mathrm{MCH}$ solution to ensure blocking of the remaining gold binding sites. Both the concentration of $\mathrm{MCH}$ and the incubation time were varied $\left(0 \mu \mathrm{M}\right.$ to $2000 \mu \mathrm{M}$ in $\mathrm{H}_{2} \mathrm{O}$ and 0 $\min$ to 120 min respectively). $\mathrm{MCH}$ was chosen due to its identical length to the $\mathrm{C}_{6}$-spacer on the DNA, which should ensure complete functionality of the DNA by forming a mixed monolayer [15].

\section{4}

\section{Raman instrumentation}

After the final surface functionalization step the chips were allowed to dry under ambient conditions in order to perform Raman measurements. Raman measurements were taken shortly after drying using a DXR ${ }^{\mathrm{TM}}$ Raman Microscope (Thermo Scientific) with the following settings: 50x optical objective (1.6 $\mu \mathrm{m}$ spot diameter), $780 \mathrm{~nm}$ excitation wavelength, $0.1 \mathrm{~mW}$ laser power and $25 \mu \mathrm{m}$ slit width within the spectral range of 500-2000 $\mathrm{cm}^{-1}$. Each sample was measured in a 30x10 point mapping configuration with $2 \mu \mathrm{m}$ step size in 
both directions. The collection time for each of the 300 mapping points was $1 \mathrm{~s}$. All maps were taken near the centre of the $4 \mathrm{~mm} \times 4 \mathrm{~mm}$ chips. The intensity of the Raman signal was manually focused before each map was collected. The raw data (without any background correction) was obtained via the OMNIC 8 software package (Thermo Scientific) and was analysed using MATLAB (MathWorks ${ }^{\circledR}$ ). All spectra shown have been offset for clarity. All data analysis was carried out on raw data.

\section{5}

\section{Data analysis model}

The use of a 2D SERS substrate enables quick gathering of large amount of data compared to traditional nanoparticle SERS. In order to analyse the entire dataset a peak-fitting model was employed on a given spectral range depending on the peak of interest. A spectrum was modelled as

$$
f(x)=\sum_{i=1}^{N_{p}} A_{i} p_{i}(x)+b(x)+\epsilon
$$

where $f(x)$ is the measured spectrum, $x$ is the Raman shift wavenumber, $p_{i}(x)$ is the peak model for the $i$ 'th peak, $A_{i}$ is the amplitude of the $i$ 'th peak, $N_{p}$ is the number of peaks, $b(x)$ is the background model and $\epsilon$ is the noise model. The peak model used in this work is a pseudo-Voigt function commonly used within vibrational spectrum modelling. It consists of a linear combination of a Lorentzian and Gaussian shaped curves defined as [16]

$$
p\left(x, x_{0}, \omega, \eta\right)=\eta L\left(x, x_{0}, \omega\right)+(1-\eta) G\left(x, x_{0}, \omega\right)
$$

where $\omega$ is the width of the peak and $0<\eta<1$ is a shape parameter. The Lorentzian curve is defined as

$$
L\left(x, x_{0}, \omega\right)=\frac{1}{1+\frac{\left(x-x_{0}\right)^{2}}{\omega^{2}}}
$$

and accounts for the response from vibrational states of a molecule. The Gaussian curve is associated with vibrational noise and is defined as

$$
G\left(x, x_{0}, \omega\right)=\exp \left(-\frac{\ln 2}{\omega^{2}}\left(x-x_{0}\right)^{2}\right)
$$

The background was modelled as a linear correction in the spectral window analysed and the error is assumed normally distributed. Prior distributions of the remaining parameters are required for Bayesian inference to be eligible. Appropriate and flexible choices are

$$
\begin{aligned}
& A \sim \Gamma\left(A_{a}, A_{b}\right) \\
& x_{0} \sim N\left(\mu_{0}, \sigma_{0}\right) \\
& \omega \sim \Gamma\left(\omega_{a}, \omega_{b}\right) \\
& \eta \sim B(1,1)
\end{aligned}
$$

where $\Gamma$ is the gamma distribution, $N$ is the normal distribution and $B$ is the beta distribution. Metropolis Hastings sampling algorithm $[17,18]$ was used for model inference. It was run with $10^{5}$ iterations and a burn-in of $5 \cdot 10^{4}$ iterations. An example of a fitted peak is shown in Supplementary information S1.

\section{3}

\section{Results and discussion}

3.1

Effect of varying DNA incubation time 
The effect of different incubation times of thiolated DNA on the gold SERS substrate was firstly investigated. After incubation in DNA solution the samples were quickly dipped twice in $\mathrm{H}_{2} \mathrm{O}$ to remove unbound molecules and prevent salt aggregation. Figure 2 shows the average $(n=300)$ spectra for varying incubation time of DNA. The figure shows a zoom of the region of observed peaks (full range spectra in Supplementary information S2). Even after only five minutes in the DNA solution a significant signal is seen. Increasing the incubation time seems to sharpen and enhance the spectrum, even though little improvement is seen after $15 \mathrm{~min}$ incubation. Each individual spectrum was subject to peak fitting in the range of $1230-1310 \mathrm{~cm}^{-1}$ where the strongest DNA peak was observed. The data is visualised in the histograms of Figure 3 where the left histogram shows the distribution of Raman intensities and the right histogram shows the distribution of the centre of the fitted peaks. For 0 min the intensity distribution is skewed towards low intensities and the centre distribution is very broad and almost uniform. This means that the model was unable to fit one particular peak at a specific wavenumber. When inspecting the spectra yielding a high intensity peak (data not shown), it became clear that some contamination from e.g. ambient air and/or salt aggregation from the buffer solution used was present on the surface. This contamination was seen to vanish when DNA and MCH was added to the buffer solution. This happens because the affinity of these molecules towards gold is much higher than that of the contaminating molecules. At 5 min incubation in the DNA solution, a distribution with a smaller width (i.e. lower standard deviation) in the peak position arises. It is still broad, but clearly centred close to $1280 \mathrm{~cm}^{-1}$ as observed in the average spectra. An interesting observation is though that the intensity distribution already at this point becomes heavy tailed towards higher intensities. By increasing the incubation time the peak position distribution becomes narrower around the $1280 \mathrm{~cm}^{-1}$ peak and the intensities becomes higher. However not much change in the histograms is seen after 30 min incubation, which tells that the surface has been fully covered with DNA at this point.

Even though a signal is seen after a short $(5 \mathrm{~min}$ ) incubation time a prolonged incubation might be more suitable. The purpose is to allow the thiol modification to form a covalent like bond to the gold, which is the rate-limiting step. When the DNA initially comes in contact with the gold nanostructure its sugar-phosphate backbone physically adsorbs onto the surface. The nitrogen in the backbone might even form weaker coordinate bonds to the gold [15]. By allowing a prolonged incubation time the formation of the intended thiol-gold bond can be obtained. Literature reports on incubation time of about 1-24 hours for strong DNAgold binding $[9,15,19,20]$. We chose 120 minutes incubation to allow sufficient time for the formation of the thiol-gold bond, even though we see no significant change in the fitted peak parameters after $30 \mathrm{~min}$. 


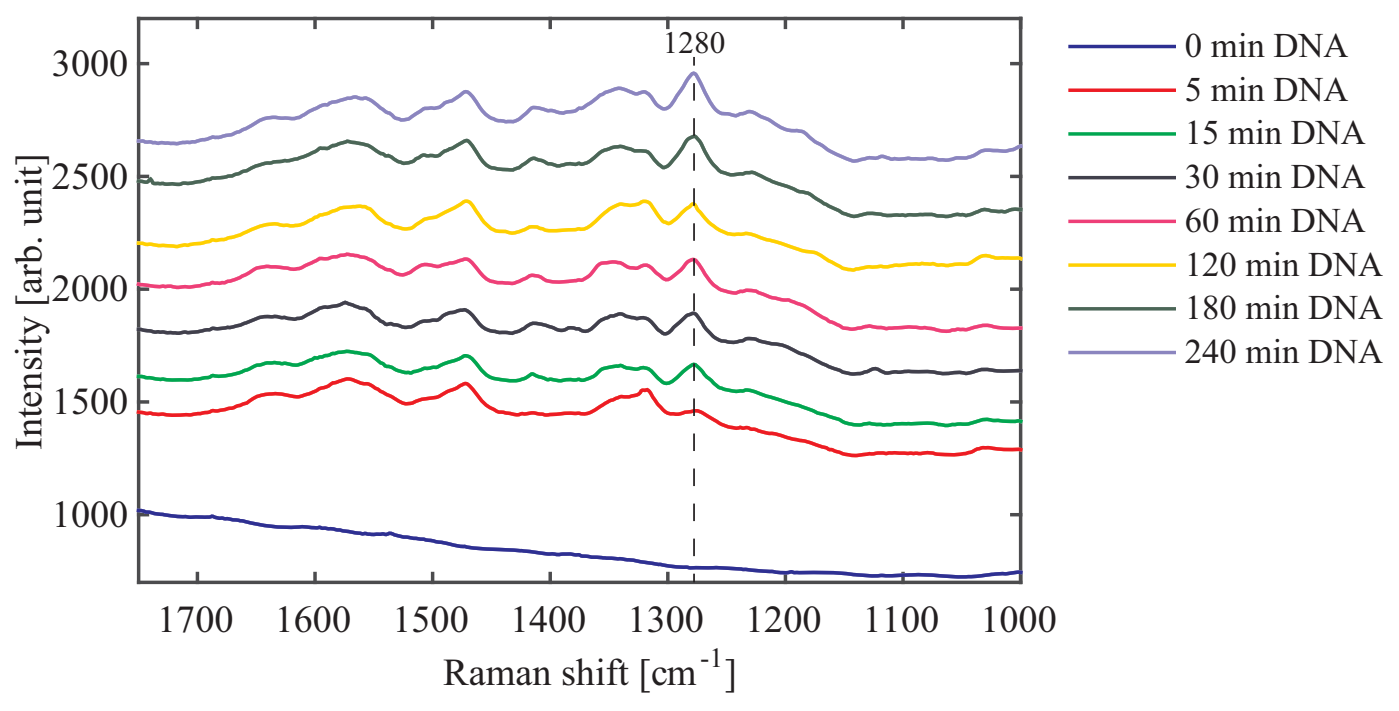

Figure 2: Average spectra of $n=300$ spectra for different incubation times of DNA. The negative control (dark blue) is clearly distinguished compared to even 5 min incubation (red) of DNA. The peak at $1280 \mathrm{~cm}^{-1}$ becomes sharper as the incubation is increased to $15 \mathrm{~min}$ (green). Further increase in incubation time shows only a minor sharpening of the $1280 \mathrm{~cm}^{-1}$ peak and no change in the general spectra. 

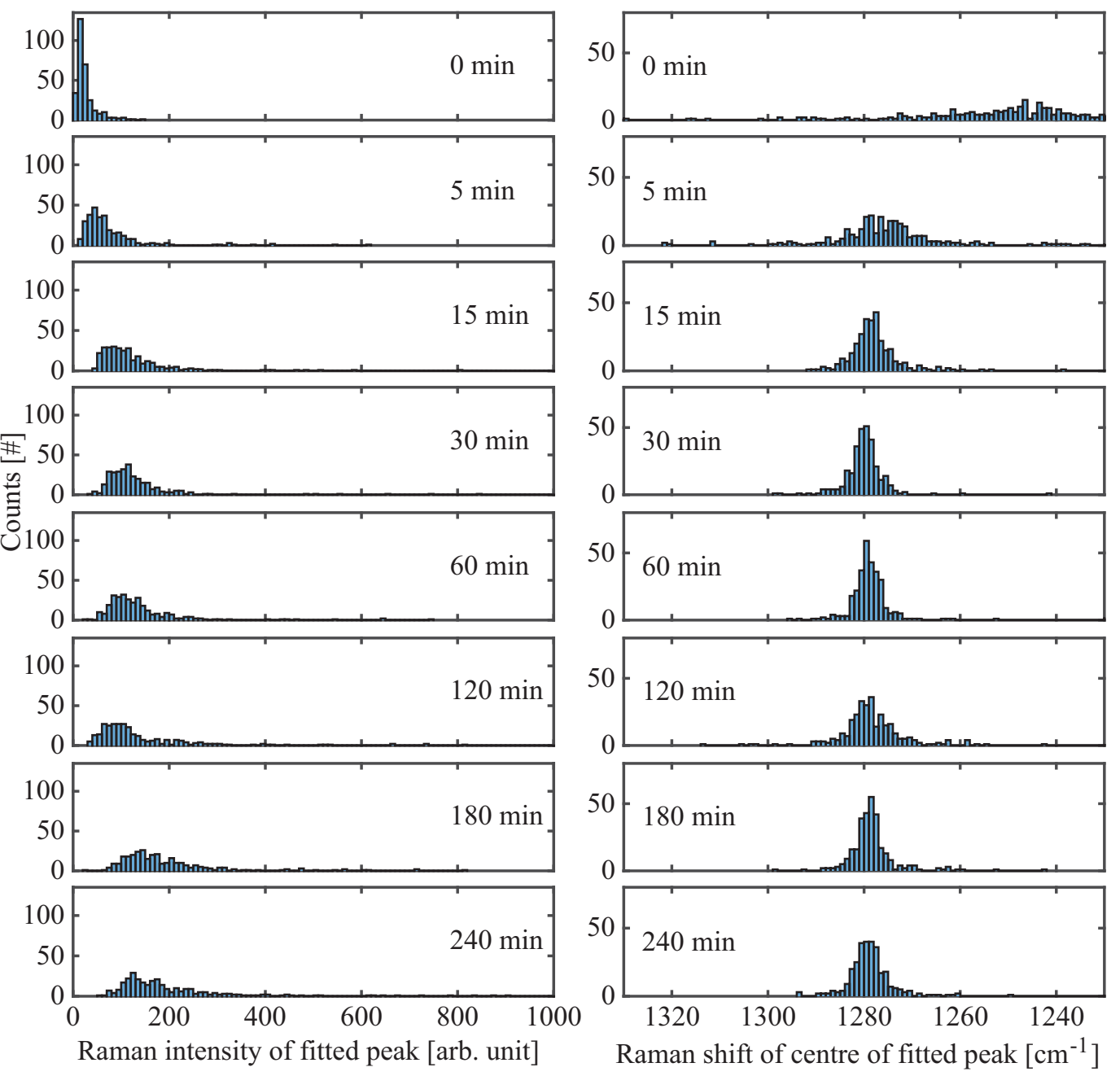

Figure 3: Histograms of fitted Raman intensity (left) and the fitted peak position (right) for varying incubation time. At $0 \mathrm{~min}$ the model struggles to fit a peak in the given range (seen by low intensities and very broad peak position distribution), but already at 5 min incubation in DNA a peak position distribution around $1280 \mathrm{~cm}^{-1}$ appears. Likewise the intensities are higher than for $0 \mathrm{~min}$ and the intensity distribution is seen to become heavy-tailed. The peak position distribution is further sharpened by increase to $15 \mathrm{~min}$ incubation, but no dramatic change is observed for the intensity.

\section{2}

\section{Effect of varying MCH concentration on DNA signal}

Secondly, the influence of $\mathrm{MCH}$ on the DNA spectra was investigated. Pure MCH shows a minor peak at $1300 \mathrm{~cm}^{-1}$ in the average spectra (Supporting information S3), which could have influence on the $1280 \mathrm{~cm}^{-1}$ DNA peak. The concentration of MCH was varied from 0-2000 $\mu \mathrm{M}$ and incubated on the DNA functionalised substrates for $60 \mathrm{~min}$. The histogram of Raman intensity and centre of fitted peaks are shown in Figure 4. Here a clear trend is observed: higher $\mathrm{MCH}$ concentrations leads to a shift in the peak position from $1280 \mathrm{~cm}^{-1}$ towards $1300 \mathrm{~cm}^{-1}$. In combination the peak intensity is seen to clearly decrease as a function of the $\mathrm{MCH}$ concentration. Inspection of the individual spectra (data not shown) reveals that the intermediate peak (e.g. at $100 \mu \mathrm{M}$ ) is in fact a poorly resolved double peak. Due to the resolution of the spectra the peakfitting model fits only one peak to this double peak. Despite this, the transition from a pure DNA signal towards an MCH dominated signal is clearly seen from the histograms. Pang et al. [20] argues that it is important to maintain the DNA peaks in the spectra (when combined with $\mathrm{MCH}$ ), in order for the DNA not 
to be relocated/removed from the surface by MCH SAMs by ligand exchange reaction. At the same time the $\mathrm{MCH}$ should also be observable in the spectra. For these reasons $50 \mu \mathrm{M}$ seems to be the most reasonable compromise between the two, which is also backed up by another MCH specific peak; namely a double peak at $1060 \mathrm{~cm}^{-1}$ and $1085 \mathrm{~cm}^{-1}$. Applying the model to fit both of these peaks in combination yields the histograms shown in Figure 5. Here is should be noted that even for very small concentrations of MCH (5-10 $\mu \mathrm{M})$ the model is able to find the double peak, which was not evident from the average spectra (Supporting information S4). At these concentrations the peak position distributions are however broad compared to higher concentrations and the Raman intensities are lower.

At $50 \mu \mathrm{M}$ the histograms shows a clear difference compared to lower concentrations in that the intensity becomes clearly divided into two different distributions, which is evidence of a double peak (with one peak being stronger than the other). Likewise the peak position distributions become sharper revealing that a more consistent peak is present in the spectra. This, in combination with the peaks fitted for the $1280 \mathrm{~cm}^{-1}$ peak, allowed for the selection of $50 \mu \mathrm{M}$ as an optimal concentration for this set of experiments.
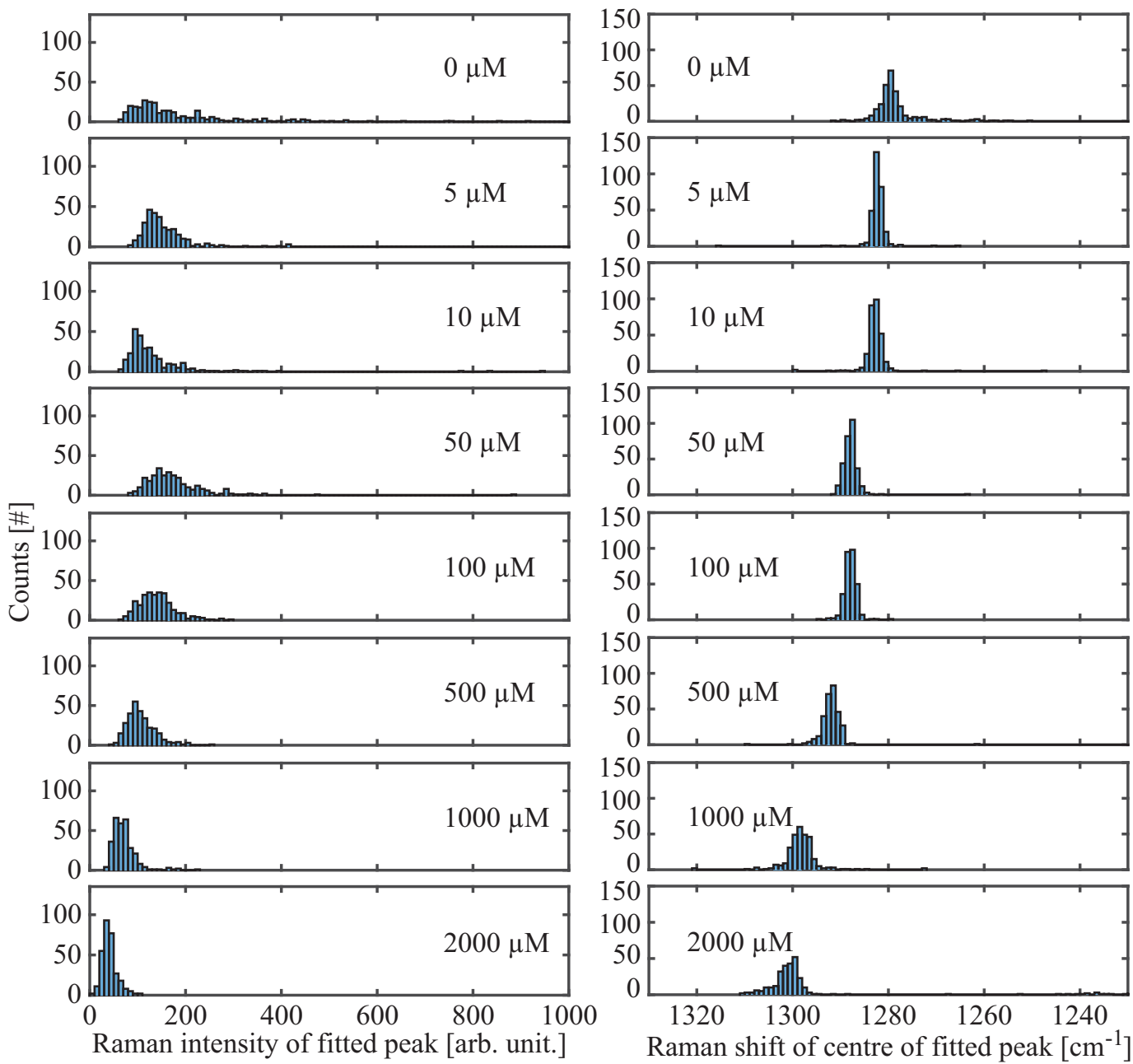

Figure 4: Histograms of fitted Raman intensities (left) and peak position (right) of the DNA peak at $1280 \mathrm{~cm}^{-1}$ for increasing $\mathrm{MCH}$ concentration. A clear trend is seen as the $\mathrm{MCH}$ concentration is increased: the peak moves towards $1300 \mathrm{~cm}^{-1}$ while the intensities are decreased. This shows that applying too much $\mathrm{MCH}$ on the nanopillars will dominate the DNA signal or even remove the DNA by a ligand exchange reaction. 

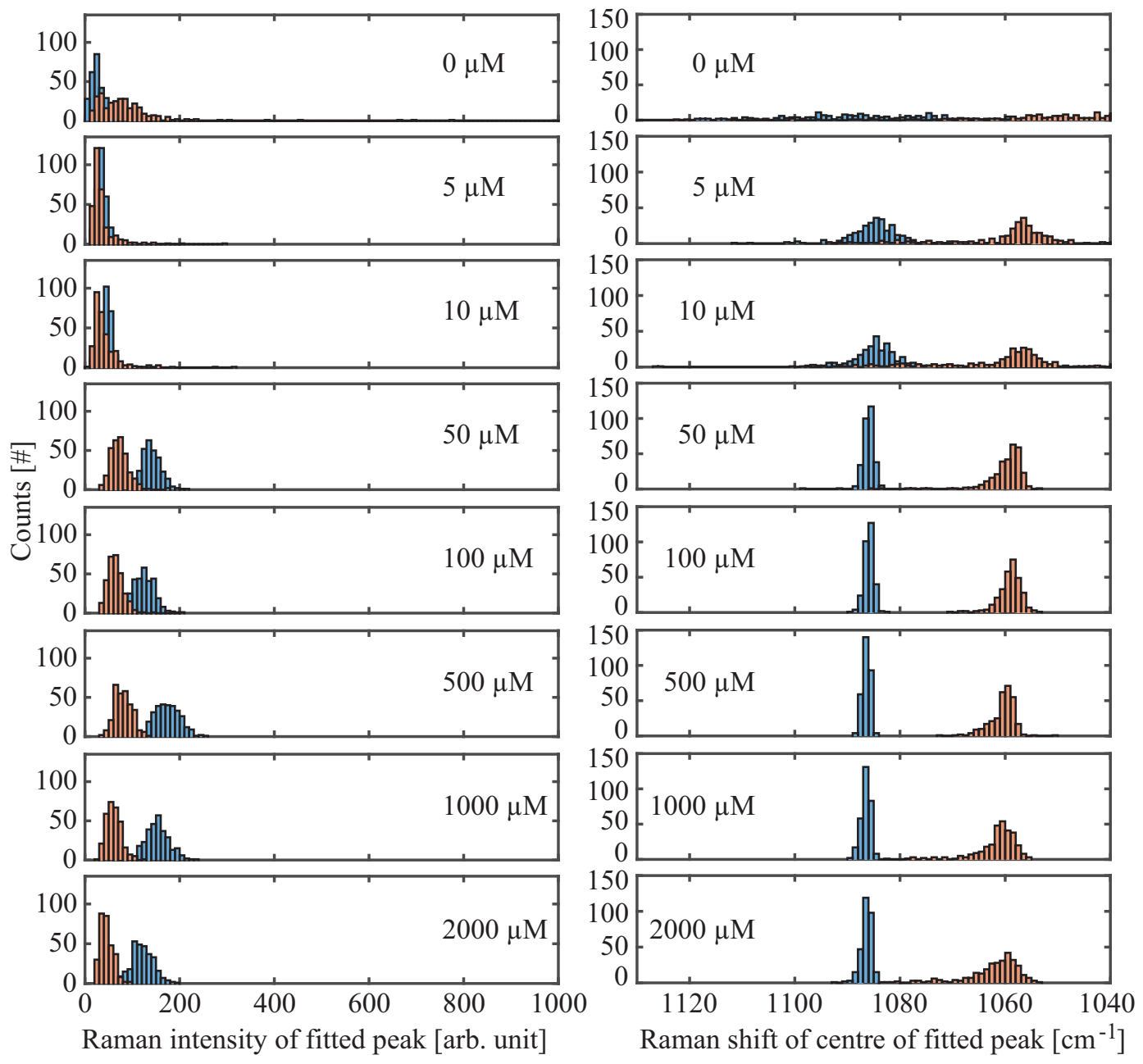

Figure 5: Histograms of fitted Raman intensities (left) and peak position (right) of the $\mathrm{MCH}$ double peak at $1085 \mathrm{~cm}^{-1}$ for increasing $\mathrm{MCH}$ concentration. It is observed that for $50 \mu \mathrm{M}$ the double peak becomes very distinct, in that the intensity distributions become separated. This is evidence of a double peak where one peak is taller than the other. At this concentration the $1280 \mathrm{~cm}^{-1}$ DNA peak in Figure 4 is only slightly shifted, which implies that the DNA is not dominated by $\mathrm{MCH}$.

\section{3}

\section{Effect of varying $\mathrm{MCH}$ incubation time}

Lastly, the effect of the MCH incubation time was investigated; the $50 \mu \mathrm{M}$ concentration was applied for 0$60 \mathrm{~min}$ and the spectra were obtained. By inspecting the histograms obtained from the peak-fitting model in Figure 6 it is seen that even very short $(5 \mathrm{~min}$ ) incubation time yields a spectra very similar to that of very long (120 min) incubation time. This reveals that $\mathrm{MCH}$ is very quick in binding to the gold surface. This very short incubation time should be suitable for $\mathrm{MCH}$ even though a covalent-like bond must be formed. This is due to the short length of the molecule and the capability of forming SAMs, which act as a seed for other molecules. 

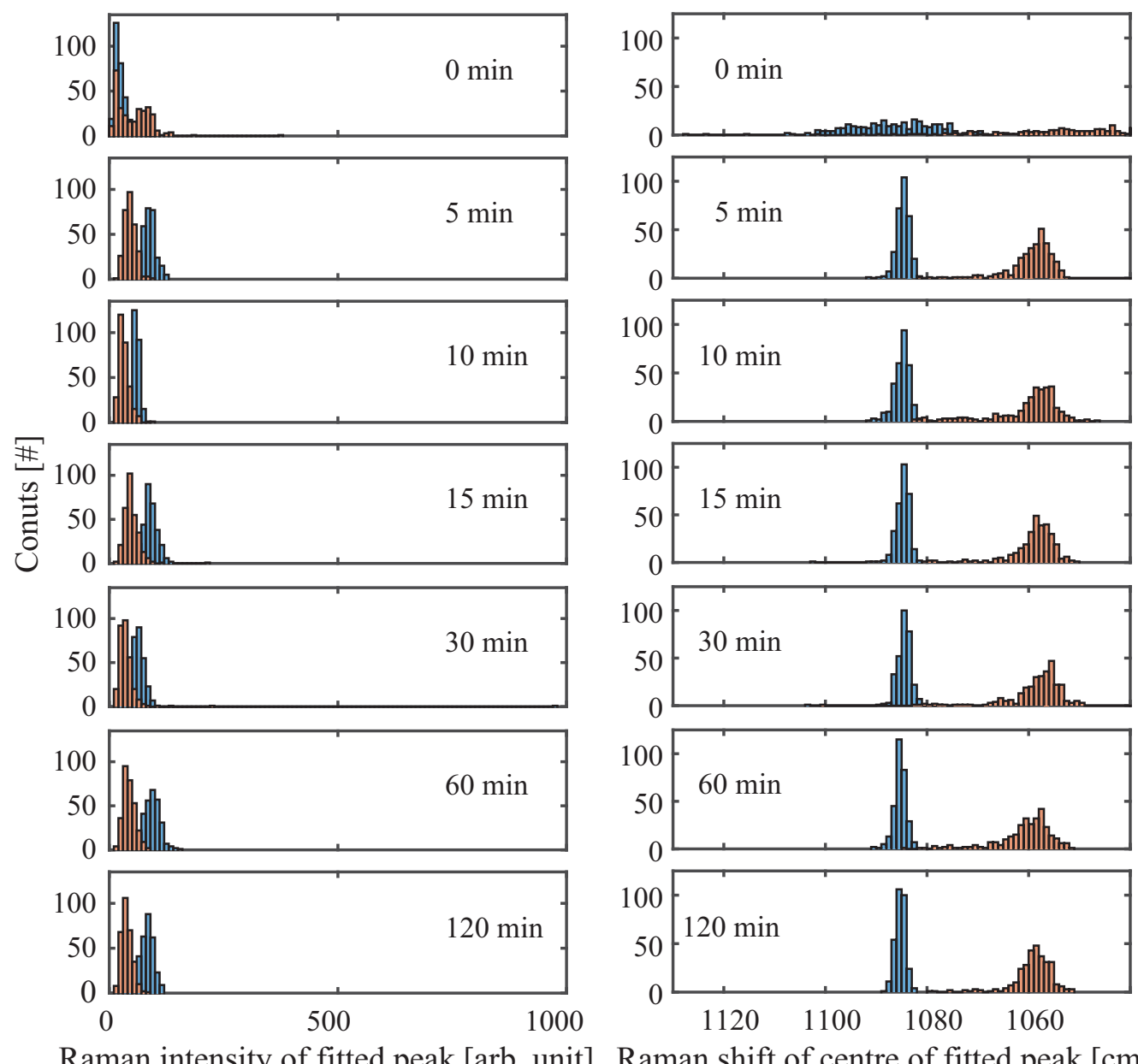

Raman intensity of fitted peak [arb. unit] Raman shift of centre of fitted peak $\left[\mathrm{cm}^{-1}\right]$

Figure 6: Histograms of fitted Raman intensities (left) and peak position (right) of the MCH double peak at $1085 \mathrm{~cm}^{-1}$ for increasing $\mathrm{MCH}$ incubation time. It is seen that 5 minutes incubation yields the same peak distributions as for 120 minutes incubation. This clearly indicates that $\mathrm{MCH}$ is quick at forming SAMs on the nanopillar surface.

\section{4}

\section{Conclusion}

We have demonstrated statistical analysis of a large amount of SERS data to directly inspect the chemical functionalization of a SERS substrate. This was achieved by employing a peak-fitting model to a specific region of the Raman spectra. By observing changes in the distribution of Raman peaks it was possible to investigate the interplay between DNA and $\mathrm{MCH}$ for varying concentrations and incubation times of $\mathrm{MCH}$. Furthermore, it confirms the classical thiol-gold reaction mechanism from studies on flat gold substrates on a nanostructured surface. This work demonstrates the strengths of statistical analysis of SERS data compared to conventional methods of obtaining only single spectra or averaging an ensemble. By using peak fitting rather than the raw data it is possible to eliminate variations due to enhanced background originating from contamination.

\section{5}

\section{Acknowledgement}

This work has been funded by the MUSE project, Innovation Fund Denmark.

\section{References}


[1] E.C. Le Ru, M. Meyer, P.G. Etchegoin, Proof of Single-Molecule Sensitivity in Surface Enhanced Raman Scattering (SERS) by Means of a Two-Analyte Technique, J. Phys. Chem. B. 110 (2006) 1944-1948. doi:10.1021/jp054732v.

[2] P.G. Etchegoin, E.C. Le Ru, A perspective on single molecule SERS: current status and future challenges., Phys. Chem. Chem. Phys. 10 (2008) 6079-6089. doi:10.1039/b809196j.

[3] S. Nie, S. Emory, Probing Single Molecules and Single Nanoparticles by Surface-Enhanced Raman Scattering, Science. 275 (1997) 1102-1106. doi:10.1126/science.275.5303.1102.

[4] K. Kneipp, Y. Wang, H. Kneipp, L.T. Perelman, I. Itzkan, R.R. Dasari, et al., Single Molecule Detection Using Surface-Enhanced Raman Scattering (SERS), Phys. Rev. Lett. 78 (1997) 1667-1670.

[5] E. Petryayeva, U.J. Krull, Localized surface plasmon resonance: Nanostructures, bioassays and biosensing - A review, Anal. Chim. Acta. 706 (2011) 8-24. doi:10.1016/j.aca.2011.08.020.

[6] B. Sharma, M. Fernanda Cardinal, S.L. Kleinman, N.G. Greeneltch, R.R. Frontiera, M.G. Blaber, et al., High-performance SERS substrates: Advances and challenges, MRS Bull. 38 (2013) 615-624. doi:10.1557/mrs.2013.161.

[7] P.G. Etchegoin, M. Meyer, E.C. Le Ru, Statistics of single molecule SERS signals: is there a Poisson distribution of intensities?, Phys. Chem. Chem. Phys. 9 (2007) 3006-3010. doi:10.1039/b704013j.

[8] C.M.S. Izumi, M.G. Mo, A.G. Brolo, Statistics on Surface-Enhanced Resonance Raman Scattering from Single Nanoshells, J. Phys. Chem. C. 115 (2011) 19104-19109. doi:10.1021/jp2068649.

[9] J. Yang, M. Palla, F. Bosco, T. Rindzevicius, T.S. Alstrøm, M.S. Schmidt, et al., Surface-Enhanced Raman Bioassay on Aptamer-Functionalized Nanopillars Using Large-Area Raman Mapping, ACS Nano. 7 (2013) 5350-5359.

[10] L.G. Carrascosa, L. Martínez, Y. Huttel, E. Román, L.M. Lechuga, Understanding the role of thiol and disulfide self-assembled DNA receptor monolayers for biosensing applications, Eur. Biophys. J. 39 (2010) 1433-1444. doi:10.1007/s00249-010-0599-6.

[11] S. Park, K. A. Brown, K. Hamad-Schifferli, Changes in oligonucleotide conformation on nanoparticle surfaces by modification with mercaptohexanol, Nano Lett. 4 (2004) 1925-1929.

doi:10.1021/n1048920t.

[12] S. Zeng, D. Baillargeat, H.-P. Ho, K.-T. Yong, Nanomaterials enhanced surface plasmon resonance for biological and chemical sensing applications., Chem. Soc. Rev. 43 (2014) 3426-3452. doi:10.1039/c3cs60479a.

[13] M.S. Schmidt, J. Hübner, A. Boisen, Large area fabrication of leaning silicon nanopillars for surface enhanced Raman spectroscopy., Adv. Mater. 24 (2012) OP11-OP18. doi:10.1002/adma.201103496.

[14] K. Wu, T. Rindzevicius, M.S. Schmidt, K.B. Mogensen, S. Xiao, A. Boisen, Plasmon resonances of Ag capped Si nanopillars fabricated using mask-less lithography, Opt. Express. 23 (2015) 1296512978. doi:10.1364/OE.23.012965.

[15] T. Herne, M. Tarlov, Characterization of DNA probes immobilized on gold surfaces, J. Am. Chem. Soc. 119 (1997) 8916-8920. doi: 10.1021/ja9719586. 
[16] G.K. Wertheim, M. A. Butler, K.W. West, D.N.E. Buchanan, Determination of the Gaussian and Lorentzian content of experimental line shapes, Rev. Sci. Instrum. 45 (1974) 1369-1371. doi:10.1063/1.1686503.

[17] W.K. Hasting, Monte Carlo sampling methods using Markov chains and their applications, Biometrica. 57 (1970) 97-109.

[18] N. Metropolis, A.W. Rosenbluth, M.N. Rosenbluth, A.H. Teller, E. Teller, Equation of State Calculations by Fast Computing Machines, J. Chem. Phys. 21 (1953) 1087-1092. doi:doi:10.1063/1.1699114.

[19] E. Chung, J. Jeon, J. Yu, C. Lee, J. Choo, Surface-enhanced Raman scattering aptasensor for ultrasensitive trace analysis of bisphenol A., Biosens. Bioelectron. 64 (2015) 560-565. doi:10.1016/j.bios.2014.09.087.

[20] S. Pang, T.P. Labuza, L. He, Development of a single aptamer-based surface enhanced Raman scattering method for rapid detection of multiple pesticides., Analyst. 139 (2014) 1895-1901. doi:10.1039/c3an02263c. 